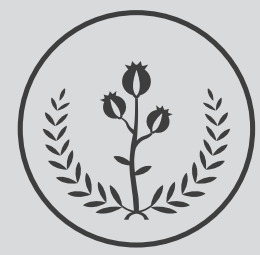

FUCS

\title{
Reacción de hipersensibilidad a ticagrelor
}

\section{Ticagrelor-induced hypersensitivity reaction}

Jorge A. Castro-Clavijo MD Laura Niño-Rojas MD $^{\mathrm{b}}$ Jhon Vivas MD ${ }^{\mathrm{c}}$

${ }^{a}$ Cardiología. Medicina Interna, Epidemiología clínica, Fundación Universitaria de Ciencias de la Salud. Bogotá DC, Colombia. ${ }^{b}$ Médico general, Fundación Universitaria de Ciencias de la Salud. Bogotá DC, Colombia.

${ }^{c}$ Medicina interna, Endocrinología, Hospital Infantil Universitario de San José, Fundación Universitaria de Ciencias de la Salud, Bogotá DC, Colombia.

\section{RES U M EN}

Introducción: el ticagrelor es un medicamento antiagregante plaquetario utilizado como prevención secundaria en pacientes con síndrome coronario agudo. Dentro de las reacciones adversas reportadas secundarias a su administración se encuentran hemorragias, cefalea, disnea, epistaxis, pausas ventriculares o bradicardia, hiperuricemia y elevación de la creatinina. No obstante, las reacciones de hipersensibilidad han sido raras. Presentación del caso: paciente masculino de 63 años con infarto agudo del miocardio, elevación de ST y documentándose en cateterismo cardíaco ectasia y enfermedad de flujos lentos. Requirió terapia de antiagregación dual con ácido acetilsalicílico (ASA) y ticagrelor, con posterior urticaria de origen medicamentoso según concepto de dermatología. Se manejó con esteroide tópico, antihistamínico oral y retiro de ticagrelor. Se considera un caso raro de reacción al antiagregante plaquetario descrito.

Palabras clave: antiagregantes plaquetarios, clopidogrel, hipersensibilidad, ticagrelor.

(C) 2022 Fundación Universitaria de Ciencias de la Salud - FUCS. Este es un artículo Open Access bajo la licencia CC BY-NC-ND (http://creativecommons.org/licenses/by-nc-nd/4.0/).

\section{INFORMACIÓN DEL ARTÍCULO}

Historia del artículo:

Fecha recibido: abril 12 de 2019

Fecha aceptado: octubre 3 de 2019
Autor para correspondencia. Dra. Laura Marcela Niño lmnino2@fucsalud.edu.co
DOI

10.31260/RepertMedCir.01217273.907 


\section{A B S T R ACT}

Introduction: ticagrelor is an antiplatelet therapy used for secondary prevention in patients with acute coronary syndrome. Adverse effects of ticagrelor are hemorrhage, headache, dyspnea, epistaxis, ventricular pauses or bradycardia, hyperuricemia and elevation of creatinine level. However, hypersensitivity reaction of ticagrelor is rarely recognized. Case report: we present a 63-year-old male patient diagnosed with acute myocardial infarction, ST-segment elevation and ectasia and low blood flow disease documented by coronary angiogram. He required dual antiplatelet therapy with acetylsalicylic acid (ASA) and ticagrelor, developing drug-induced urticaria according to dermatology consultation. He was treated with topical steroids, oral antihistamines and ticagrelor discontinuation. This case is considered a rare case of reaction to the antiplatelet agent described herein.

Key words: antiplatelet drugs; clopidogrel; hipersensitivity; ticagrelor.

(C) 2022 Fundación Universitaria de Ciencias de la Salud - FUCS. This is an open access article under the CC BY-NC-ND license (http://creativecommons.org/licenses/by-nc-nd/4.0/).

\section{INT RODUCCIÓN}

El ticagrelor es uno de los nuevos antiagregantes plaquetarios aprobado para uso y distribución comercial por la FDA en 2011. Actúa como antagonista selectivo no competitivo, sin bioactivación hepática y que proporciona un bloqueo reversible del receptor P2Y12 de adenosin difosfato (ADP) en la superficie de las plaquetas, lo cual lo diferencia del clopidogrel que inhibe de manera irreversible (unión covalente) el receptor y es un profármaco, por lo tanto implica dos vías de metabolismo hepático. ${ }^{1}$

Respecto a las reacciones adversas, la principal se caracteriza por hemorragia (tabla1); ${ }^{2}$ sin embargo, la hipersensibilidad al medicamento según la FDA se ha reportado con incidencia de $10 / 1000$ personas, es decir, entre los pacientes que se encuentran en manejo con ticagrelor $0.9 \%$ puede presentar esta reacción, ${ }^{3}$ con primer caso reportado en 2014 por Quinn y Connelly en Canadá. ${ }^{4}$

De esta manera, en el presente reporte de caso documentamos un evento raro de reacción de hipersensibilidad a dicho medicamento, con pocos informes a nivel mundial. ${ }^{5}$ En los casos leves el diagnóstico ha sido solo clínico, salvo un caso de reacción severa con dermatosis neutrofílica febril aguda que requirió biopsia. ${ }^{3}$ El primer caso publicado mostró infiltrado linfocítico. ${ }^{4}$

Tabla 1. Reacciones adversas frecuentes a ticagrelor|

\begin{tabular}{|l|c|} 
Disnea & $13.8 \%$ \\
Hemorragia & $11.6 \%$ \\
Cefalea & $6.5 \%$ \\
Epistaxis & $6 \%$ \\
Hiperuricemia y elevación de creatinina & $\mathrm{ND}^{*}$ \\
Hipersensibilidad & $\mathrm{ND}^{*}$ \\
\hline
\end{tabular}

${ }^{*} \mathrm{ND}$ : no hay porcentajes claros reportados. Fuentes: los autores.

\section{REPORTE DE CASO}

Paciente masculino de 63 años que consulta al Hospital Infantil Universitario de San José de Bogotá el 23 de enero de 2018 por cuadro clínico de 3 horas de evolución, caracterizado por dolor torácico irradiado a cuello y miembro superior izquierdo, más síntomas neurovegetativos y electrocardiograma en el que se evidencia infarto agudo del miocardio con supradesnivel del ST en cara inferior. Se realiza trombólisis con criterios de reperfusión y angiografía coronaria que documenta oclusión distal de la arteria descendente posterior, flujos lentos y ectasia coronaria. El ecocardiograma transtorácico revela cardiopatía isquémica, fracción de eyección del ventrículo izquierdo conservada en $55 \%$, signos indirectos de hipertensión pulmonar y dilatación biauricular, sin angina posinfarto y evolución favorable. El servicio de cardiología indica manejo de tipo antiagregación dual con ácido acetilsalicílico (ASA) y ticagrelor, además de IECA, betabloqueador y estatina, dando egreso temprano.

No obstante, el paciente consultó 5 horas después por aparición de prurito intenso y eritema en dorso de manos y pies, que se extendió con habones a región cervical, torácica, lumbar y luego a las extremidades (figuras 1 y 2), que dermatología conceptuó como urticaria de origen medicamentosa y determinó administración de corticoide tópico, antihistamínico oral y suspensión del ticagrelor, ante la sospecha de ser el causante de hipersensibilidad, con favorable evolución clínica. Se da egreso de nuevo formulando ASA y clopidogrel, con mejoría de las lesiones en piel y resolución del cuadro clínico a las 3 semanas, atribuyéndose el evento al ticagrelor, ya que los demás medicamentos descritos no fueron descontinuados en ningún momento. Días después, se cita a control por consulta externa en donde se evidencia paciente asintomático, con adecuada evolución clínica. 


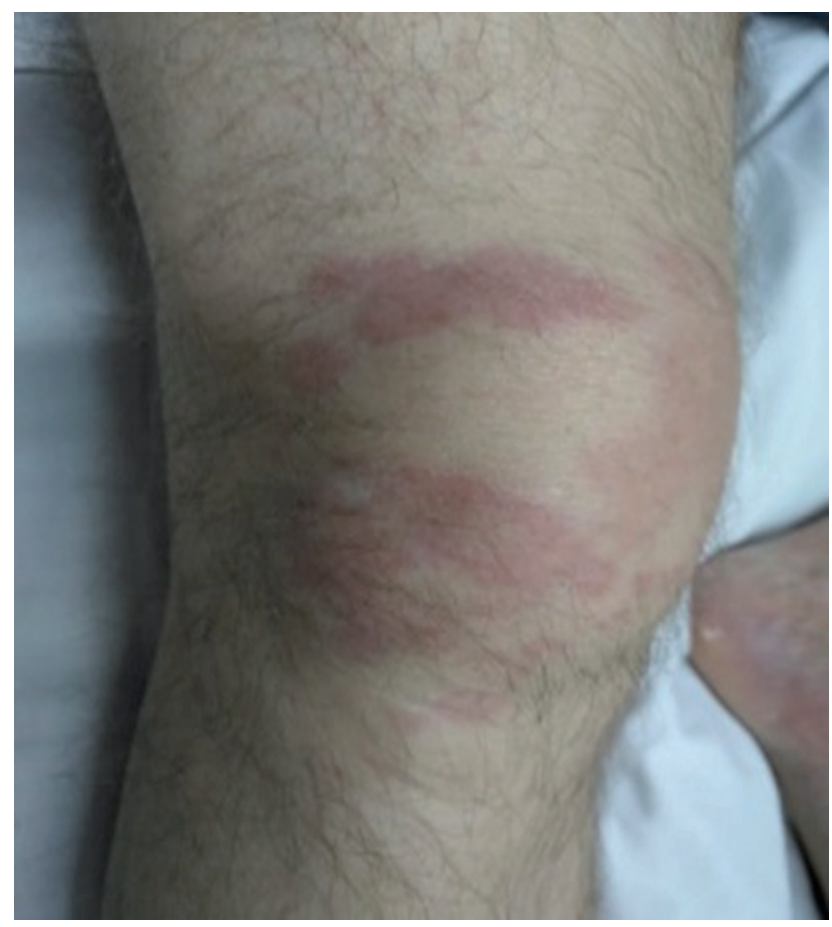

Figura 1. Imagen de lesiones iniciales del paciente. Fuente: los autores.

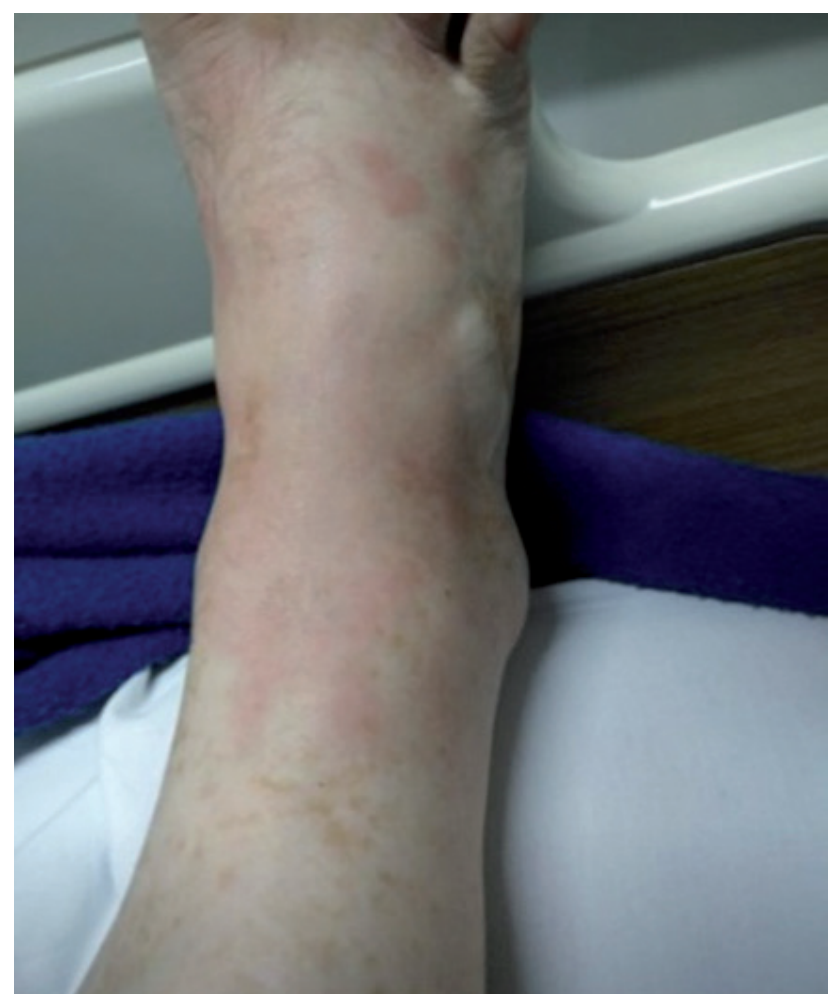

Figura 2. Extensión de lesiones iniciales. Fuente: los autores.

\section{ISCUSIÓN}

Los antiagregantes plaquetarios a pesar de compartir el mismo mecanismo de acción se dividen en dos grupos de acuerdo con su estructura química, encontrando las tienopiridinas (clopidogrel y prasugrel) y las ciclopentiltriazolopirimidinas (ticagrelor y cangrelor), ${ }^{6}$ que según la literatura difieren en las reacciones adversas que puedan llegar a presentarse. La reacción cruzada de hipersensibilidad es menos probable entre los dos grupos, sin embargo puede ocurrir, considerándola como no secundaria a reacción inmunológica sino por compartir el mismo mecanismo de acción. $^{7}$

En la literatura se ha documentado en reportes de casos, que los pacientes que desarrollaron alergia a ticagrelor no tenían antecedente documentado de reacción secundaria a otros medicamentos durante su vida, como se observó en este caso. ${ }^{4,7}$ Así mismo, la sintomatología y la presentación del cuadro clínico es variable desde rash maculopapular con prurito, habones, dermatosis neutrofílica febril aguda o incluso angioedema, con predominio de tronco y menos en extremidades, similar al presente caso.,

En general los casos se han manejado con corticoides tópicos, antihistamínicos y retiro del ticagrelor, con lo cual hay disminución de la alergia. Además, en la mayoría se ha optado por cambio a clopidogrel resolviéndose la sintomatología, manteniéndose así la prevención secundaria. No obstante, en caso de que se presente reacción con otro de los nuevos antiagregantes ya nombrados, una buena alternativa es la desensibilización o incluso el uso de otros medicamentos, incluidos los anticoagulantes orales, que tienen menor evidencia clínica en dicho contexto. ${ }^{5}$

Es de importancia clínica conocer las reacciones adversas poco frecuentes de algunos medicamentos, en especial los antiagregantes plaquetarios que en el contexto de síndrome coronario agudo son el pilar del manejo. Los reportes de caso nos pueden dar pautas para el diagnóstico y tratamiento.

CONFLICTO DE INTERESES

Los autores declaran no tener ningún conflicto de intereses. 


\section{REFERENCIAS}

1. Badimon L, Mendieta G, Vilahur G. Diferencias en los mecanismos de acción de los nuevos antiagregantes: ¿cómo actúan?. Rev Esp Cardiol Supl. 2014;14:3-9.

2. Centro Andaluz de Documentación e Información de Medicamentos. Informes de evaluación de medicamentos. Ticagrelor España: Centro Andaluz de Documentación e Información de Medicamentos (CADIME); 2013. p. 52.

3. Ikram S, Veerappan Kandasamy V. Ticagrelor-induced Sweet Syndrome: an unusual dermatologic complication after percutaneous coronary intervention. Cardiovasc Interv and Ther. 2017;32(3):244-6. doi: 10.1007/s12928-016-0398-9.

4. Quinn KL, Connelly KA. First report of hypersensitivity to ticagrelor. Can J Cardiol. 2014;30(8):957 e3-4. doi: 10.1016/j. cjca.2014.04.011.

5. Dai J, Lyu S, Ge C. Hypersensitivity to ticagrelor and low response to clopidogrel: a case report. Asia Pacific allergy. 2017;7(1):51-3. doi: 10.5415/apallergy.2017.7.1.51.

6. Jacobson KA, Boeynaems JM. P2Y nucleotide receptors: promise of therapeutic applications. Drug discovery today. 2010;15(1314):570-8. doi: 10.1016/j.drudis.2010.05.011.

7. Chin N, Rangamuwa K, Mariasoosai R, Carnes J, Thien F. Oral antiplatelet agent hypersensitivity and cross-reactivity managed by successful desensitisation. Asia Pac Allergy. 2015;5(1):51-4. doi: 10.5415/apallergy.2015.5.1.51. 